\title{
Cultural and genetic transmission in the Jomon-Yayoi transition examined in agent-based demographic simulation
}

\author{
Naoko Matsumoto \\ Graduate School of Humanities and Social Sciences, \\ Okayama University, Japan \\ Email: naoko_m@cc.okayama-u.ac.jp
}

\author{
Mariko Sasakura \\ Department of Computer Science, \\ Okayama University, Japan \\ Email: sasakura@momo.cs.okayama-u.ac.jp
}

\section{Motivation}

The transition from the Jomon to the Yayoi period of Japanese Archipelago is an East Asian case of hunter-gatherer to farmer transitions. Drastic socio-cultural changes in subsistence, material culture and settlement structure occurred in the northern part of Kyushu Island around 10th -8th centuries BC. Major drive of this transition has been attributed to either immigrants from the Korean Peninsula or intentional adoption by native Jomon people. In reality, the Jomon-Yayoi transition is a complex process in which both human migration and cultural transmission played a major role.

Simulation studies can be very useful to understand the nature of the transition as we can examine various hypotheses with different parameters to see what combination would fit archaeological data. However, simulation researches have not developed for this prehistoric event except for a series of publications by Takahiro Nakahashi and Masaru Iizuka ([1], [3], [4]). Nakahashi and lizuka suggested that the immigrant people were mainly responsible for the drastic cultural change based on palaeodemographic simulation in which higher population growth rate was assumed for immigrants. Nakahashi and Iizuka's studies demonstrate that even minor number of immigrants from the Korean Peninsula can lead to the dominance of continental physical features in the Middle Yayoi population in northern Kyushu as indicated by excavated skeletal remains.

Their model may provide a possible scenario concerning population dynamics in northern Kyushu, but its assumptions on demographic processes may be too simple to understand the real dynamics of intermarriage and resulting genetic spread, and cultural transmission process is out of its scope. Our research is the first application of agent based simulation to the Jomon-Yayoi transition with specific interest in the cultural transmission process in a realistic demographic dynamics.

The purpose of our research is not to replicate the actual processes of the Jomon-Yayoi transition in terms of genetic and cultural transmission, but to obtain useful insights to construct a model to explain the prehistoric demographic and cultural dynamics. We have considerably rich archaeological data thanks to the huge number of excavations most of which have been conducted by governmental institutions as a form of rescue archaeology. As for the Final Jomon to the Early Yayoi period, which is the critical period to understand the JomonYayoi transition, more than two hundred sites have been excavated only in Fukuoka prefecture in northern Kyushu.

\section{JOMON-YAYOI TRANSITION}

The richness of archaeological information tell us that the actual process of the transition, i.e., the nature and extent of migration, adoption or rejection of cultural information, etc., varied from region to region even in western Japan, where it has once been suggested that the spread of Yayoi culture was quick and uniform. Interpretation of archaeological evidence also differs among researchers due to different assumptions concerning migration, cultural transmission, and the relationship between the two. As it is extremely difficult to calculate the long-term consequences of particular assumptions on genetic and cultural transmission, simulation study can be a very useful tool to experiment with particular set of assumptions.

Critical points of the Jomon-Yayoi transition which have been reconstructed based on archaeological data can be summarized as follows:

1) There was a certain amount of migration from the Korean Peninsula to northern Kyushu at the beginning of the Yayoi period [3].

2) Yayoi culture was formed by integrating traditional Jomon culture and Korean Early Bronze Age culture in northern Kyushu [2].

3) Major components of the Yayoi culture in northern Kyushu consist of wet rice agriculture, Itazuke type (Ongagawa-style) pottery, polished stone tools similar to the Korean Bronze Age culture, and new burial customs similar to that of the Korean Bronze age.

4) Immigrants from the Korean Peninsula and indigenous group of the Jomon tradition lived in the same settlements in northern Kyushu.

5) The spread of Yayoi culture to the other part of western Japan was achieved probably by both migration and 
acculturation, but their actual conditions are still not clear.

6) Several cases of the discovery of skeletal remains with Jomon features accompanied by Yayoi cultural traits indicate that indigenous adoption of new cultural elements was not rare.

7) Some of the cultural elements such as placement of stone weapon as burial goods were dropped in the process of diffusion from northern Kyushu to Chugoku and Kinki regions [5].

8) Wet rice agriculture and Ongagawa-style pottery spread from the northern Kyushu to Chugoku and Kinki regions across about $500 \mathrm{~km}$ keeping considerable uniformity.

As pointed out above, the nature and extent of migration from the northern Kyushu to the east, and how different patterns of migration and acculturation may result in the distribution of genes and cultural traits, have not been well understood. Our simulation project intends to gain basic understanding of the relationships between the migration rate and the spread of genetic traits, and how social learning condition affect the spread of cultural skill in a realistic demographic dynamics.

\section{Simulation System}

The simulation system we developed is a multi-agent system. In the system, an individual is implemented as an agent. They are born, married, giving birth, and died according to calculated rates of these events would be happened. Population increase or decrease can be controlled to some extent by adjusting birth rate and death rate, although the results can be varied because of the stochastic nature of the simulation.

The system has areas that represent geographical divisions, or regional boundary of population. Areas are connected, and the connections of these areas can be logically represented as a graph in which an area is a node and a connection between areas is an edge. Individuals are born in one of the areas and can move through the connections between areas, if she or he decides to move. The system determines if the migration occurs for each individual according to the migration rate.

An individual represented as an agent in the system is born as a female or a male with $50 \%$ probability and has knowledge about her/his blood relationship, so that we can set incestuous taboo. An agent start finding her/his spouse at the age of 15 , and females can give birth to babies only when she is married. Both female and male remarry when her/his spouse dies.

Each agent has two parameters: genetic value and skill value. The genetic value g represents a set of genes responsible for the morphological difference between the Jomon and the Yayoi phenotypes. The skill value represents the level of cultural skill like production of material culture.

Assume a population of $\mathrm{N}$ individuals, $i=1,2, \ldots, N$. Each has genetic value $g_{i}$ and skill value $s_{i}$.

$g_{i}$ is defined at the birth of $i$-individual according to the genetic value of parents. If the parents of $i$-individual are $j$ and $k, g_{i}=\left(g_{j}+g_{k}\right) / 2$. $s_{i}$ is 0 at the birth of $i$-individual. If $i$-dividual can grow up to be 15 years old, the $i$-individual choose her/his master $m$ and copies the value of $s_{m}$ to $s_{i}$. The system has three choice of the master to simulate the effect of social learning in a simplified manner. One of them is selected when the simulation runs.

L1. Choose randomly from all individuals in the same area who are more than 15 years old.

L2. Choose one with the highest skill value among relatives who live in the same area.

L3. Choose one with the highest skill value in the same area.

Here, relatives are individuals within the 3 degree of relationship.

In order to obtain insights about genetic influence and cultural transmission in the Jomon-Yayoi transition, we set 5 areas connected in series, as area A, B, C, D and E respectively. 300 individuals are generated in each area at the start of simulation, and data is taken after 30 years when population structure is normalized. At the start of the simulation, $g_{i}$ of each individual who lives in area $\mathrm{A}$ is 1 and that of those who live in other areas is $0 . s_{i}$ of the individuals living in area $\mathrm{A}$ is set according to normal distribution with a mean of 10.0 and sigma of 1.0. $s_{i}$ of other areas are 0.0 . We experimented with three sets of migration rate.

M1. An individual moves in 0.001 possibility per year.

M2. An individual moves alone in 0.03 possibility per year.

M3. An individual moves with her/his relatives within the 2 degree of relationship in 0.001 possibility per year.

Each individual can move to a neighboring area, that is, if an individual lives in area $\mathrm{A}$, she/he moves to area $\mathrm{B}$ in a specified possibility. If an individual lives in area $\mathrm{B}$, she/he moves to area $\mathrm{A}$ or $\mathrm{C}$ in a specified possibility for each year.

We ran five simulations for 500 years for each of the three versions of cultural transmission pattern and examined the results of population increase, spread of genetic value and spread of skill value. Birth rate and death rate were set as the population gradually increases.

\section{RESUlts}

Results of five runs are shown overlapped in the graphs so that basic patterns and variability can be assessed. The rate of population increase shows considerable variation (Fig. 1) . The smallest population at the end of simulation for 500 years is less than 2000 in some cases while the largest is over 10000. However, patterns of the spread of genetic value and skill value seem unaffected by such difference of population increase. The spread of genetic value is almost constant as the same marriage rules and move rate were applied to all runs (Fig. 2-4), while the spread of skill value showed definitely different patterns according to the ways to chose her/his master.

In the L1 condition of random selection, the skill spread very slowly (Fig. 5). Individuals who acquire skill value appear in the area B soon but only appear in the area $\mathrm{C}$ after about 100 years. The mean of skill values learned at the age of 15 slightly increases in the areas B and C while that in the area A gradually decreases. 
In the $\mathrm{L} 2$ condition of leaning from the most skilled person among relatives, skill spreads much quicker, and the level of skill does not drop during the diffusion process. Individuals with skill appear in the most distant area E around 200 years after the start even in the M1 condition (Fig. 6). In the L3 condition of the extremely biased transmission, the graphs show similar pattern with that of L2 condition but the time needed for the skill to reach area $\mathrm{E}$ is about the half.

The number of people who move during the first 200 years does not differ very much between the M2 and M3 conditions, although the number of moving people per year shows much more variability in the M3 condition. Both gvalue and skill spread slightly quicker in the M2 condition (Fig. 3, 4, 7, 8) .

\section{CONClusion}

The following insights were gained from the analysis:

1) Genetic influence and culturally transmitted skill show clearly different patterns.

2) Cultural skill can spread quickly without much loss in the case of biased transmission, even when migration rate is very small.

3) The rate of population increase can considerably vary due to chance factors.

4) Nonrandom migration based on family relationship produce results different from those of random migration in the spread of gvalue and skill.

The result provides us with a possible explanatory model for the Jomon-Yayoi transition, where indigenous people are considered to have played more major roles in the areas farther from the northern Kyushu where Yayoi culture was born under the influence of immigrants from the Korean Peninsula. How to choose her/his master may be different according to the nature of cultural skill. For highly visible, easily transmitted type of skill, an extremely biased transmission as simulated here as case 3 may be possible. However, for the kind of skills that need long time apprenticeship is necessary, people tend to learn the skill from nearby relatives. It is expected that this type of cultural skill would spread much slower than more easily acquirable elements. However, in our simulation with L3 condition in which an agent learn from the most skilled relative within the 3 degree of relationship who live in the same area, the speed of the spread of skill was not significantly different from the results with $\mathrm{L} 2$ condition. We need to further examine the effect of social learning types on the spread of cultural skill, taking the nature of kinship structure and gender into consideration.

It should be noted that migration rate, birth rate and death rate are constant for all agents in the current setting, in order to examine the relationship between variables. In the case of the Jomon-Yayoi transition, migration could be more restricted and the movement was one-way. Although our simulation project is still at preliminary stage, further examination and comparison with detailed archaeological data would produce more sound results.

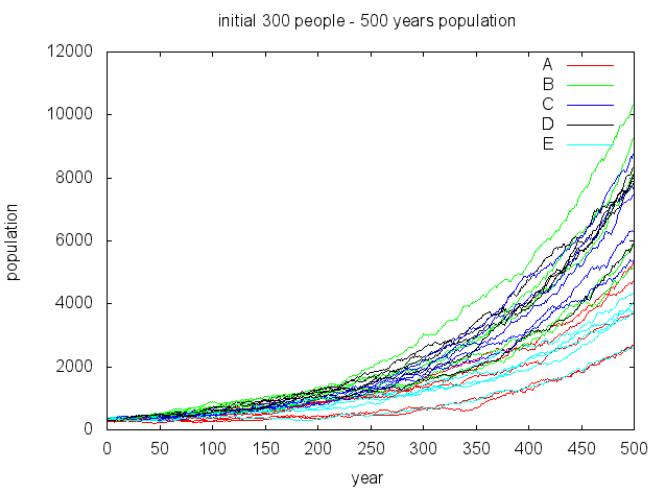

Fig. 1. Population increase: M3.

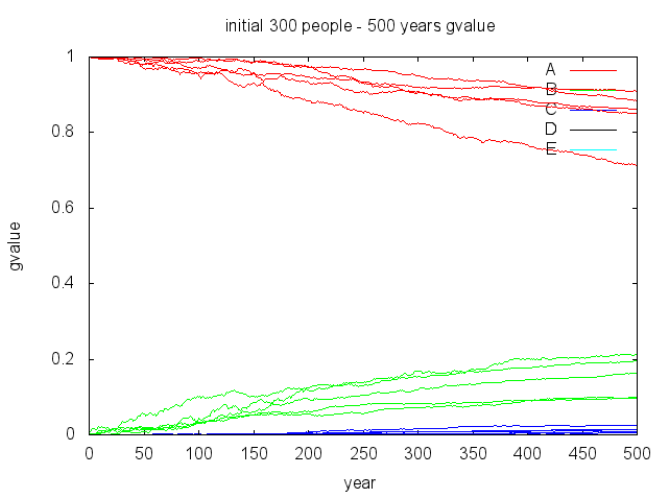

Fig. 2. Spread of gvalue: M1.

\section{REFERENCES}

[1] Iizuka, M. and Nakahashi, T. "A population genetic study on the transition from Jomon to Yayoi people," Genes. Genet. Syst., 77, 287-330, 2002.

[2] Matsumoto, N. "The Jomon-Yayoi transition and ethnicity," Quarterly of Archaeological Studies, Vol. 49(2), 2002 (in Japanese).

[3] Nakahashi, T. and Iizuka, M. "Anthropological study of the transition from the Jomon to the Yayoi periods in the northern Kyushu using morphological and palaeodemographical features (1)," Anthropological Science (Japanese Series) Vol. 106(1), 31-53. 1998 (in Japanese).

[4] Nakahashi, T. and Iizuka, M. "Anthropological study of the transition from the Jomon to the Yayoi periods in the northern Kyushu using morphological and palaeodemographical features (2)," Anthropological Science (Japanese Series) Vol. 116(2), 131-143. 2008 (in Japanese).

[5] Nakamura, D. "The diversity of mortuary practice acceptance at the beginning of the Yayoi Period," In Matsumoto, N., Bessho, H. and Tomii, M. (eds.), Coexistence and Cultural Transmission in East Asia, pp. 223256. Left Coast Press, Walnut Creek, 2011. 
Miguel, Amblard, Barceló \& Madella (eds.) Advances in Computational Social Science and Social Simulation Barcelona: Autònoma University of Barcelona, 2014, DDD repository <http://ddd.uab.cat/record/125597>

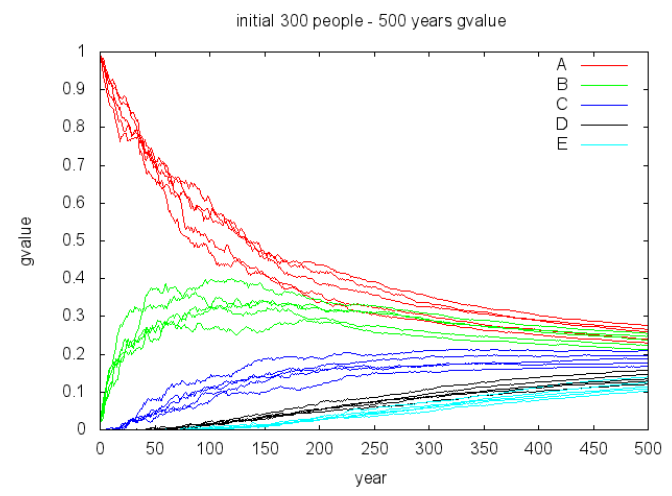

Fig. 3. Spread of gvalue: M2.

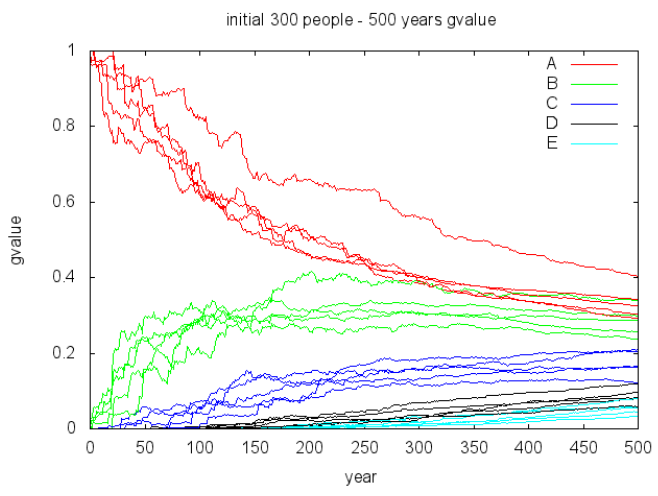

Fig. 4. Spread of gvalue: M3.

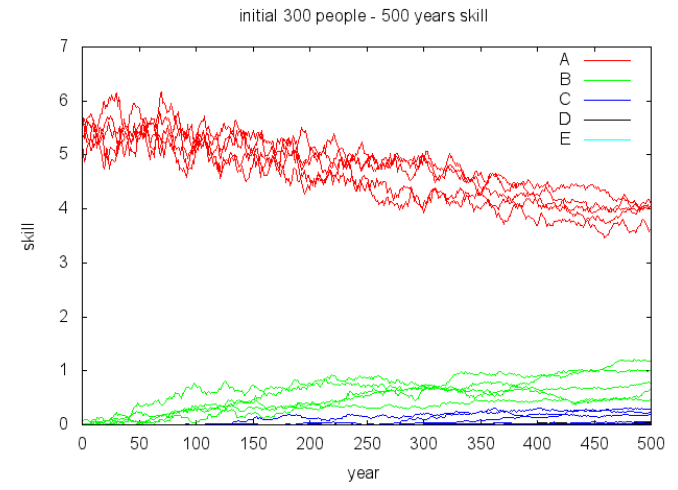

Fig. 5. Spread of skill: M1 L1.

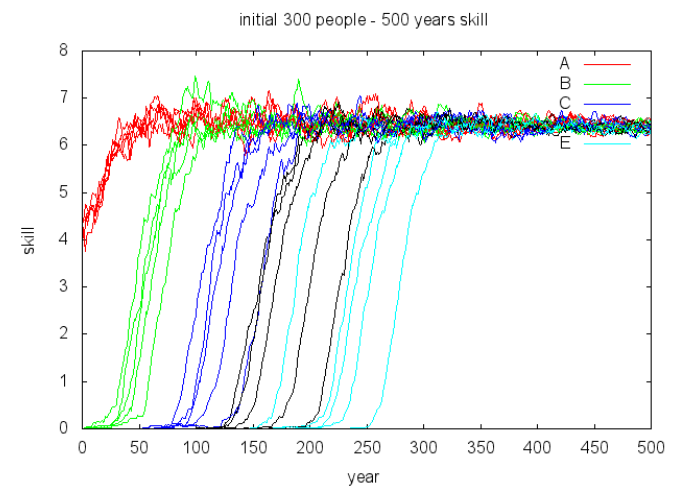

Fig. 6. Spread of skill: M1 L2.

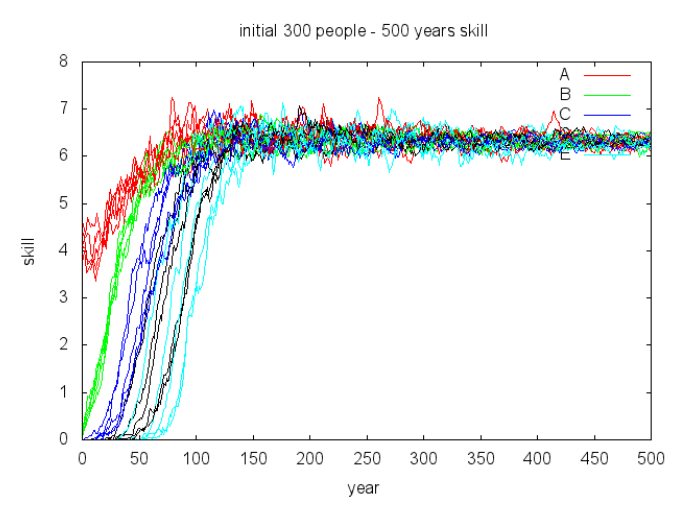

Fig. 7. Spread of skill: M2 L2.

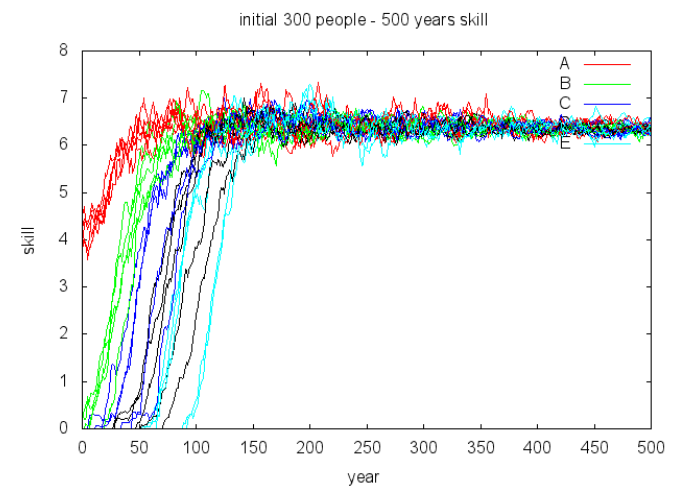

Fig. 8. Spread of skill: M3 L2. 